\title{
Loss of trefoil factor 1 is regulated by DNA methylation and is an independent predictive factor for poor survival in advanced gastric cancer
}

\author{
TOMOKAZU TANAKA ${ }^{1}$, JUN NAKAMURA ${ }^{1}$, YOSHIHIKO KITAJIMA ${ }^{1,2}$, KEITA KAI $^{3}$, SHUUSUKE MIYAKE ${ }^{1}$, \\ MASATSUGU HIRAKI ${ }^{1}$, TAKAO IDE ${ }^{1}$, YASUO KOGA ${ }^{1}$ and HIROKAZU NOSHIRO ${ }^{1}$ \\ ${ }^{1}$ Department of Surgery, Saga University Faculty of Medicine, Saga 849-8501; ${ }^{2}$ Department of Surgery, \\ National Hospital Organization Higashisaga Hospital, Saga 849-0101; ${ }^{3}$ Department of Pathology \\ and Microbiology, Saga University Faculty of Medicine, Saga 849-8501, Japan
}

Received October 9, 2012; Accepted November 30, 2012

DOI: $10.3892 /$ ijo.2013.1759

\begin{abstract}
Trefoil factor 1 (TFF1) is considered to be a tumor suppressor gene in gastric cancer. However, the role of TFF1 expression and its regulation in gastric cancer patients remain unclear. The aims of this study were to clarify the clinical significance of TFF1 and to determine its regulatory mechanisms. We assessed the immunohistochemical expression of TFF1 in 182 gastric cancer patients and examined whether or not TFF1 is associated with the clinicopathological factors and patient survival. In vitro study using TFF1 knockdown gastric cancer cells evaluated the role of TFF1 in cancer invasion. Bisulfite sequencing was performed to assess DNA methylation of TFF1 in cells and resected tissues. Patients with low expression of TFF1 showed a significantly deeper invasion of the tumor than those with high expression $(\mathrm{p}=0.037)$. Low expression of TFF1 was also associated with a poor survival $(\mathrm{p}=0.029)$ in 108 patients who were treated by surgery alone. Both TFF1 expression and lymph node metastasis are independent predictive factors for disease-specific survival in a multivariate analysis. In an in vitro study, invasive power of the cells was significantly increased in the TFF1-deficient cells compared with the control cells. Bisulfate sequencing showed that TFF1 expression is strongly dependent on DNA methylation in both gastric cancer cells and tissues. Interestingly, methylation status of two specific CpG sites, which are located close to a TATA box and hypoxia response element (HRE), determined the TFF1 expression in the resected tissues. TFF1 expression is silenced by DNA methylation and is associated with tumor invasion and a poor survival in gastric cancer patients. The expression and/or methylation
\end{abstract}

Correspondence to: Dr Jun Nakamura, Department of Surgery, Saga University Faculty of Medicine, 5-1-1 Nabeshima, Saga 849-8501, Japan

E-mail: nakamurj@cc.saga-u.ac.jp

Key words: trefoil factor 1, pS2, gastric cancer, epigenetic modification, survival status of TFF1 may, therefore, serve as a useful biomarker for predicting survival in patients with advanced gastric cancer.

\section{Introduction}

The trefoil factor (TFF) family is a group of protease-resistant peptides characterized by a conserved three-loop domain, designated as a TFF domain (1-3). The TFF family consists of pS2 (TFF1), spasmolytic polypeptide SP (TFF2) and intestinal trefoil factor (TFF3) (4), which are all widely expressed in the gastrointestinal epithelium. TFF1 was initially described as an estrogen-inducible gene in the hormone-sensitive breast cancer cell line MCF-7 (5), but was later found to be spontaneously expressed at a high level in gastric epithelial cells (6). TFF1 interacts with mucin core proteins to stabilize the surface mucus gel layers of the gastric epithelium and plays an important role in the protection and regeneration of the gastric mucosa $(2,7,8)$. On the contrary, TFF1-deficient mice develop antral neoplasms of the stomach (9), thus suggesting that TFF1 acts as a tumor-suppressor gene in the stomach. Moreover, several investigators have reported that TFF1 expression is related to carcinogenesis and metastasis in various types of cancers, such as prostate, breast, pancreatic and upper gastrointestinal cancer (10-14).

The mechanisms regulating TFF1 expression were previously addressed. It has been demonstrated that several similar sequences at the 5'-flanking regions of human TFF genes (motifs I-IV) play an important role in the stomach-specific expression of TFF1 $(15,16)$. GATA- 6 activates TFF1 expression in the stomach via motif III (17) and HNF-3 activates TFF1 transcription via motif IV, located close to the TATA box (18). Hernández et al (19) also indicated that hypoxia inducible factor (HIF)-1 mediates the induction of TFF1 expression in gastric epithelial cells under hypoxic conditions. However, few studies have so far examined the clinical significance of TFF1 expression and its regulatory mechanism in gastric cancer patients.

The present study evaluated the immunohistochemical expression of TFF1 in 182 gastric cancer patients and examined whether or not TFF1 is associated with the clinicopathological factors and patient survival. In addition, to clarify 
the mechanism regulating TFF1 expression, we focused on the methylation status of the TFF1 promoter region.

\section{Materials and methods}

Patients. One hundred and eighty-two patients with advanced gastric cancer who underwent curative surgery at our institution between June 2000 and December 2008 were enrolled. None of these patients had hepatic, peritoneal, or distant metastasis, nor any tumor cells in the peritoneal fluid. The stage classification was performed according to the guidelines of the Japanese Gastric Cancer Association (20). Informed consent to use specimens was obtained from all patients and the study protocol was approved by the Ethics Committee of Saga University Faculty of Medicine.

Cell culture. Eight gastric cancer cell lines (MKN1, MKN7, MKN28, MKN45, MKN74, HSC45, HSC57 and KATO-III) were used for the study. HSC45 and HSC57 were provided by Dr K. Yanagihara (National Cancer Center Research Institute, Tokyo, Japan) $(21,22)$ and the remaining six cell lines were purchased from Cell Bank, RIKEN BioResource Center (Ibaraki, Japan). The cells were maintained under either normoxic conditions (containing 20\% $\mathrm{O}_{2}$ and $5 \% \mathrm{CO}_{2}$ in air) or hypoxic conditions (containing $1 \% \mathrm{O}_{2}, 5 \% \mathrm{CO}_{2}$ and $94 \% \mathrm{~N}_{2}$ ).

Immunohistochemistry. Paraffin-embedded sections (4- $\mu \mathrm{m}$-thick) were incubated with an anti-TFF1 antibody (1:100 dilution; sc-28925, Santa Cruz Biotechnology, Santa Cruz, $\mathrm{CA}$ ) for $2 \mathrm{~h}$ at room temperature and with the corresponding secondary antibody for $30 \mathrm{~min}$. The slides were washed in PBS and incubated with a diaminobenzidine substrate kit (Nichirei Corp., Tokyo, Japan). The level of cytoplasmic staining for TFF1 was classified as high expression and low expression, according to the percentage of stained cancer cells. Tumors showing $30 \%$ or more positive cancer cells were considered to have high expression, while $<30 \%$ staining was judged as the low expression.

Total-RNA extraction and real-time RT-PCR. RT-PCR was performed using the Light Cycler instrument system (Roche Diagnostics GmbH, Mannheim, Germany) according to the manufacturer's instructions. The primers were designed as follows: TFF1, 5'-TTGTGGTTTTCCTGGTGTCA-3', 5'-GGGACG TCGATGGTATTAGG-3' (111 bp) and $\beta$-actin, 5'-TTAAGG AGAAGCTGTGCTACG-3', 5'-GTTGAAGGTAGTTTCGTG GAT-3' (206 bp). A melting curve analysis was used to control for the specificity of the amplification products. The quantitative value was normalized to the $\beta$-actin expression level, which was used as an internal control.

DNA extraction and bisulfite sequencing. The frozen tissue specimens from six representative patients were cut into $8 \mu \mathrm{m}$-thick sections and cancer foci were macrodissected. Genomic DNA from gastric cancer cell lines and macrodissected cancer foci obtained from gastric cancer tissues was extracted using a DNeasy Blood \& Tissue kit (Qiagen, Hilden, Germany). Bisulfite modification was then carried out using an EpiTect Plus Bisulfite kit (Qiagen) with 500 ng of genomic
DNA. Bisulfite-specific primers were designed as follows: 5'-TTTGTTTTGGTTTTTTAAAGTGTTG-3', 5'-TTCTCC ATAATAACCATTACCTCCT-3' (611 bp) and 5'-GAGGAG GTAATGGTTATTATGGAGA-3', 5'-AAACCCTACCAC CCTAAATTACTCT-3' (283 bp). Bisulfite PCR was performed using the Takara ExTaq Hot Start Version (Takara, Shiga, Japan). The PCR products were purified using a QIAquick PCR Purification kit (Qiagen) and then ligated into the pT7Blue-T-vector (Novagen, Madison, WI) using a DNA Ligation kit Ver.2.1 (Takara). E. coli DH5 $\alpha$ competent cells (Toyobo Co., Ltd., Osaka, Japan) were transformed by inserting the vector described above and were seeded on LB plates containing the appropriate antibiotics and incubated overnight at $37^{\circ} \mathrm{C}$. For each sample, at least 10 clones were sequenced by a dye terminator method on an Applied Biosystems 3130 Genetic Analyzer (Applied Biosystems, Inc., Foster City, CA) using a BigDye Terminator v3.1 Cycle Sequencing kit (Applied Biosystems). The results were analyzed using the Sequence Scanner Software program, v1.0 (Applied Biosystems).

Knockdown of TFF1. siRNA oligos for the human TFF1 gene (SASI_Hs01_00121167) and non-silencing (scrambled) siRNA (siRNA Universal Negative Control \#1) were purchased from Sigma-Aldrich, Inc. The siRNA oligos were transiently transfected into HSC45 and HSC57 cells using a MicroPorator-mini (MP-100) (Digital Bio Technology, Seoul, Korea) in combination with the Neon ${ }^{\mathrm{TM}} 100-\mu 1$ kit (Invitrogen Corp., Carlsbad, $\mathrm{CA}$ ), according to the manufacturer's instructions. The transfected cells were cultured in complete medium and used on 1 to 4 days of culture. Western blot analysis confirmed that the silencing effect on the gene expression continued for $144 \mathrm{~h}$ after the transfection.

Western blot analysis. Whole-cell lysates from cultured cells were prepared using lysis buffer as described in a previous report (23). Aliquots containing $50 \mu \mathrm{g}$ of protein were subjected to 4 to $12 \%$ Bis-Tris gel electrophoresis (NuPAGE; Invitrogen) and were transferred onto Amersham Hybond-ECL membranes (GE Healthcare, Buckinghamshire, UK) in transfer buffer. After blocking with 5\% skim milk for $60 \mathrm{~min}$, the membranes were incubated with primary antibodies overnight at $4^{\circ} \mathrm{C}$. The primary antibodies were anti-TFF1 $(1: 1000$ dilution; 2801-1, Epitomics, Burlingame, CA) and anti- $\beta$-actin (1:10,000 dilution; Sigma-Aldrich, Inc.). After incubation with the corresponding secondary antibodies, the signals were developed using an Amersham ECL Prime Western Blotting Detection System (GE Healthcare).

In vitro cell invasion assay. Polycarbonate filters $(6.5-\mathrm{mm}$ diameter, $8-\mu \mathrm{m}$ pore size) of the Falcon Transwell ${ }^{\mathrm{TM}}$ chemotaxis chambers (Becton-Dickinson, Franklin Lakes, NJ) were coated with $50 \mu \mathrm{l}(1 \mathrm{mg} / \mathrm{ml})$ of Matrigel biomatrix (Becton-Dickinson) in cold RPMI-1640 medium and dried overnight. Suspensions of $1 \times 10^{5}$ (HSC45) or $5 \times 10^{5}$ (HSC57) cells in $200 \mu \mathrm{l}$ of complete RPMI-1640 medium were placed in the upper compartments of the chamber, whereas the lower compartments were filled with $800 \mu \mathrm{l}$ of conditioned medium from MRC5 fibroblasts. These culture units were incubated for $48 \mathrm{~h}$ at $37^{\circ} \mathrm{C}$ under normoxia. The number of viable invasive 
Table I. Correlation between TFF1 expression and clinicopathological features.

TFF1 expression

\begin{tabular}{|c|c|c|c|}
\hline \multirow{2}{*}{ Characteristics } & & \multirow[b]{2}{*}{ p-value } \\
\hline & High $(n=94)$ & Low $(\mathrm{n}=88)$ & \\
\hline Age $($ mean $\pm \mathrm{SD})$ & $68.0 \pm 11.2$ & $68.4 \pm 12.3$ & 0.824 \\
\hline \multicolumn{4}{|l|}{ Gender } \\
\hline Male & 61 & 59 & \multirow[t]{2}{*}{0.760} \\
\hline Female & 33 & 29 & \\
\hline \multicolumn{4}{|l|}{ Histology } \\
\hline Differentiated & 42 & 30 & \multirow[t]{2}{*}{0.144} \\
\hline Undifferentiated & 52 & 58 & \\
\hline \multicolumn{4}{|l|}{$\mathrm{T}^{\mathrm{a}}$} \\
\hline 2 & 31 & 17 & \multirow[t]{2}{*}{0.037} \\
\hline $3 / 4$ & 63 & 71 & \\
\hline \multicolumn{4}{|l|}{$\mathrm{N}^{\mathrm{b}}$} \\
\hline- & 32 & 33 & \multirow[t]{2}{*}{0.627} \\
\hline+ & 62 & 55 & \\
\hline \multicolumn{4}{|l|}{$1 y^{c}$} \\
\hline- & 22 & 16 & \multirow[t]{2}{*}{0.463} \\
\hline+ & 72 & 72 & \\
\hline \multicolumn{4}{|l|}{$v^{d}$} \\
\hline- & 54 & 42 & \multirow[t]{2}{*}{0.212} \\
\hline+ & 40 & 46 & \\
\hline \multicolumn{4}{|l|}{ Stage } \\
\hline I/II & 54 & 38 & \multirow[t]{2}{*}{0.054} \\
\hline III & 40 & 50 & \\
\hline \multicolumn{4}{|c|}{ Adjuvant chemotherapy } \\
\hline- & 57 & 51 & \multirow[t]{2}{*}{0.764} \\
\hline+ & 37 & 37 & \\
\hline
\end{tabular}

${ }^{\mathrm{a}} \mathrm{T}$, depth of cancer invasion; ${ }^{\mathrm{b}} \mathrm{N}$, lymph node metastasis; ${ }^{\mathrm{c} l y}$, lymphatic invasion; ${ }^{\mathrm{d}} \mathrm{v}$, vascular invasion.

cells, which had infiltrated onto the lower surface of the filter, was counted.

Cell proliferation assay. Cell proliferation was analyzed by the MTT assay using a Cell-Titer $96^{\mathrm{TM}}$ non-radioactive cell proliferation assay kit (Promega, Madison, WI). In brief, $1 \times 10^{3}$ (HSC45) or $5 \times 10^{3}$ (HSC57) cells per well were seeded in triplicate onto 96 -well plates and incubated at $37^{\circ} \mathrm{C}$ in a humidified atmosphere. After $24 \mathrm{~h}$, the numbers of viable cells were measured in triplicate every day for four days. The proliferation curves were then constructed by calculating the mean value of the optical density measurements at $590 \mathrm{~nm}$ using a 96-well plate reader (Immuno-mini NJ2300, Nalge Nunc International K.K., Tokyo, Japan).

Statistical analysis. The statistical analysis was performed using the computer software program SPSS 15.0J for windows (Chicago, IL). The $\chi^{2}$ test was used to compare categorical data and differences in the mean values were evaluated by Student's t-test and the Mann-Whitney $U$ test. Both the univariate analysis and multivariate analysis for survival were performed using Cox's proportional hazards model. The survival curves were generated using the Kaplan-Meier method and the statistical significance of differences was compared using the log-rank test. Values of $\mathrm{p}<0.05$ were considered to be statistically significant.

\section{Results}

Patient characteristics. The 182 patients included 120 males and 62 females, ranging from 26 to 91 years old (median, 67.9 years). The median follow-up period was 46.3 months (range, 0.3-120.0 months). Among the 182 patients, 74 received 5-FU-based adjuvant chemotherapy (adjuvant group), while the remaining 108 did not receive adjuvant treatment (surgery group) because of the diagnosis of early stage disease, advanced age and/or various complications.

Immunohistochemical staining of TFF1. The staining of TFF1 was predominantly distributed in the cytoplasm. High expression of TFF1 (Fig. 1A) was observed in 94 gastric cancer patients, whereas the remaining 88 patients showed low expression (Fig. 1B). Low expression of TFF1 was significantly correlated with deeper invasion of the tumor $(p=0.037)$ (Table I). However, no significant correlations were observed between the TFF1 expression and other factors including age, 
A

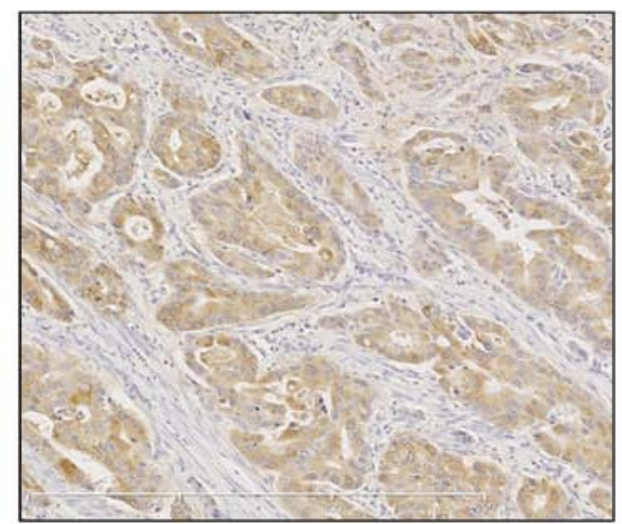

B

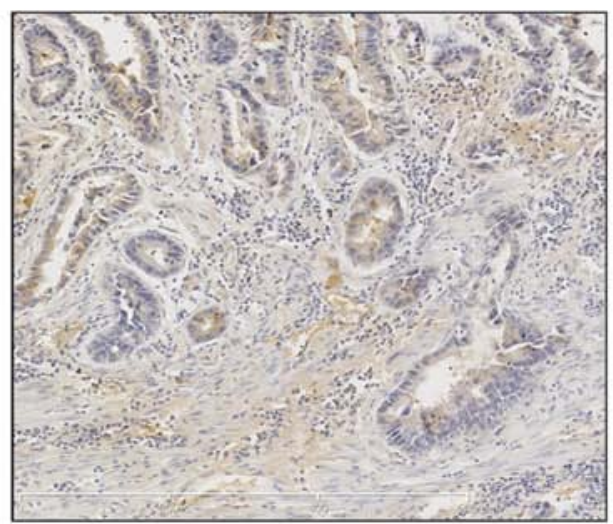

C All patients $(\mathrm{n}=182)$

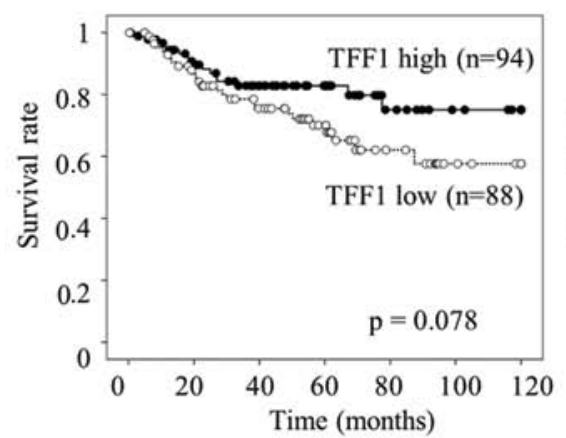

D Surgery group $(n=108)$

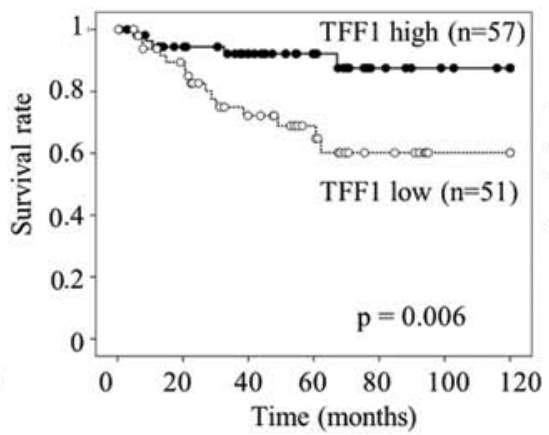

E Adjuvant group $(\mathrm{n}=74)$

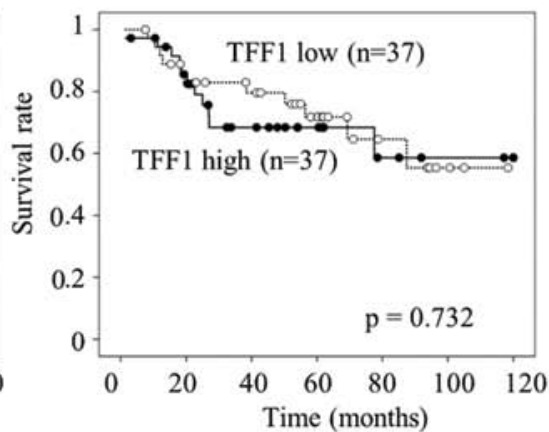

Figure 1. Immunohistochemical analysis of TFF1 expression (magnification x400) and patient survival. Expression of TFF1 was predominantly found in cytoplasma of cancer cells. (A) The upper left panel shows a case with high expression and (B) the upper right panel shows a case with low expression. Kaplan-Meier estimates of the disease-specific survival (C) in all patients, (D) in the surgery group and (E) in the adjuvant group.

A

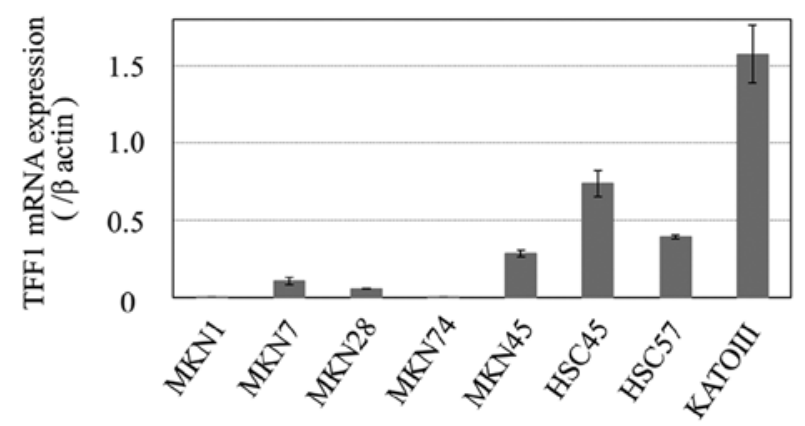

B

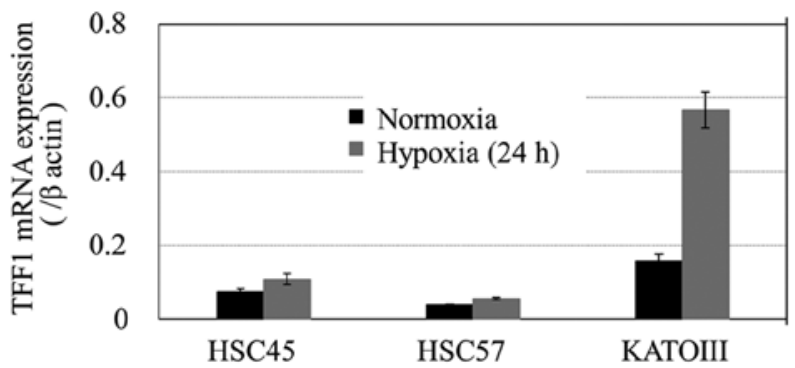

Figure 2. Real-time quantitative RT-PCR analyses of the TFF1 expression under normoxic and hypoxic conditions. (A) Under normoxic conditions, high expression of TFF1 was observed in HSC45, HSC57 and KATO-III cells, while the low expression was in MKN1, MKN28 and MKN74 cells. (B) The expression of TFF1 under hypoxic conditions was investigated after exposure to hypoxia for 24 h. The hypoxic induction of TFF1 was detected in HSC45 and KATO-III, but not in HSC57.

gender, lymph node metastasis $(\mathrm{N})$, lymphatic invasion (ly), vascular invasion (v), or tumor stage.

TFF1 expression and patient survival. In all the 182 patients, the patients with a low expression of TFF1 tended to have a worse survival than those with a high expression $(\mathrm{p}=0.078$, Fig. 1C). A low expression of TFF1 significantly associated with a worse survival in the surgery group ( $p=0.006$, Fig. 1D), whereas the TFF1 expression did not contribute to the patient survival in the adjuvant group $(\mathrm{p}=0.732$, Fig. 1E). A univariate analysis demonstrated that the depth of tumor invasion (T), lymph node metastasis (N), lymphatic invasion (ly), tumor stage and TFF1 expression were significantly associated with the disease-specific survival in the surgery group. A multivariate analysis confirmed both TFF1 expression and lymph node metastasis to be independent predictive factors 
Table II. Univariate and multivariate analysis for disease-specific survival in 108 patients in the surgery group.

\begin{tabular}{|c|c|c|c|c|c|}
\hline \multirow[b]{2}{*}{ Characteristics } & \multirow[b]{2}{*}{ No. } & \multicolumn{2}{|c|}{ Univariate } & \multicolumn{2}{|c|}{ Multivariate } \\
\hline & & HR $(95 \%$ CI $)$ & $\mathrm{p}$-value & HR (95\% CI) & $\mathrm{p}$-value \\
\hline TFF1 & & & 0.010 & & 0.026 \\
\hline High & 57 & 1 & & 1 & \\
\hline Low & 51 & $3.774(1.370-10.417)$ & & $3.268(1.151-9.259)$ & \\
\hline Age (years) & & & 0.411 & & \\
\hline$<70$ & 43 & 1 & & & \\
\hline$\geq 70$ & 65 & $1.473(0.585-3.704)$ & & & \\
\hline Gender & & & 0.828 & & \\
\hline Male & 74 & 1 & & & \\
\hline Female & 34 & $1.107(0.442-2.778)$ & & & \\
\hline Histology & & & 0.750 & & \\
\hline Differentiated & 50 & 1 & & & \\
\hline Undifferentiated & 58 & $1.157(0.470-2.849)$ & & & \\
\hline $\mathrm{T}^{\mathrm{a}}$ & & & 0.016 & & 0.266 \\
\hline 2 & 43 & 1 & & 1 & \\
\hline $3 / 4$ & 65 & $6.061(1.404-26.316)$ & & $2.353(0.521-10.638)$ & \\
\hline $\mathrm{N}^{\mathrm{b}}$ & & & 0.001 & & 0.009 \\
\hline- & 58 & 1 & & 1 & \\
\hline+ & 50 & $27.027(3.636-200.000)$ & & $16.393(2.041-142.857)$ & \\
\hline $1 y^{c}$ & & & 0.029 & & 0.386 \\
\hline- & 33 & 1 & & 1 & \\
\hline+ & 75 & $9.434(1.266-71.429)$ & & $2.494(0.316-19.608)$ & \\
\hline$v^{d}$ & & & 0.344 & & \\
\hline- & 65 & 1 & & & \\
\hline+ & 43 & $1.527(0.635-3.676)$ & & & \\
\hline Stage & & & 0.001 & & \\
\hline $\mathrm{I} / \mathrm{II}$ & 78 & 1 & & & \\
\hline III & 30 & $13.158(4.367-40.000)$ & & & \\
\hline
\end{tabular}

${ }^{\mathrm{a}} \mathrm{T}$, depth of cancer invasion; ${ }^{\mathrm{b}} \mathrm{N}$, lymph node metastasis; ${ }^{\mathrm{c} l y}$, lymphatic invasion; ${ }^{\mathrm{d}} \mathrm{v}$, vascular invasion.

for disease-specific survival $(\mathrm{p}=0.026,0.009$, respectively) (Table II).

Correlation between promoter methylation and the expression of TFF1. The expression of TFF1 mRNA was detected in the order of KATO-III > HSC45 > HSC57 > MKN45 > MKN7 $>$ MKN28 $>$ MKN74 $>$ MKN1 (Fig. 2A). Based on the results, we classified the cell lines into a high-expression group (KATO-III, HSC45 and HSC57) and a low-expression group (MKN1, MKN28 and MKN74). The hypoxia-mediated induction of mRNA was evaluated in the high-expression group and the degree of the induction was observed in the order of KATO-III > HSC45 > HSC57 (Fig. 2B).

A diagram of the promoter region of the TFF1 gene is shown in Fig. 3A. There are $22 \mathrm{CpG}$ sites between 274 and -469 bp. Fig. 3B indicates the methylation status of each $\mathrm{CpG}$ site in the six cell lines. The cell lines classified into the low-expression group showed dense methylation; in contrast, the cell lines in the high-expression group showed sparse methylation. In sharp contrast, in cancer tissues with low TFF1 expression, two specific CpG sites, which are located at -457 and -20 from the transcript initiation site, showed a significantly denser methylation in comparison to the high-expression tissues (Fig. 3C).

Characterization of TFF1 knockdown cells. TFF1 knockdown study was performed in order to investigate the role of TFF1 in cell proliferation and invasion using gastric cancer cell lines HSC45 and HSC57 with TFF1 positive expression. The TFF1 knockdown by siRNA was validated by a western blot analysis (Fig. 4A). As demonstrated in Fig. 4B, the invasive capacity was significantly increased in the TFF1 knockdown cells compared with the control cells, while there were no significant differences in the proliferation curves between the knockdown and control cells (Fig. 4C).

\section{Discussion}

The loss of TFF1 expression in gastric mucosa leads to the initiation of gastric cancer (9). However, it has been under discussion whether changes in TFF1 expression might affect the progression of gastric cancer. The present study showed 


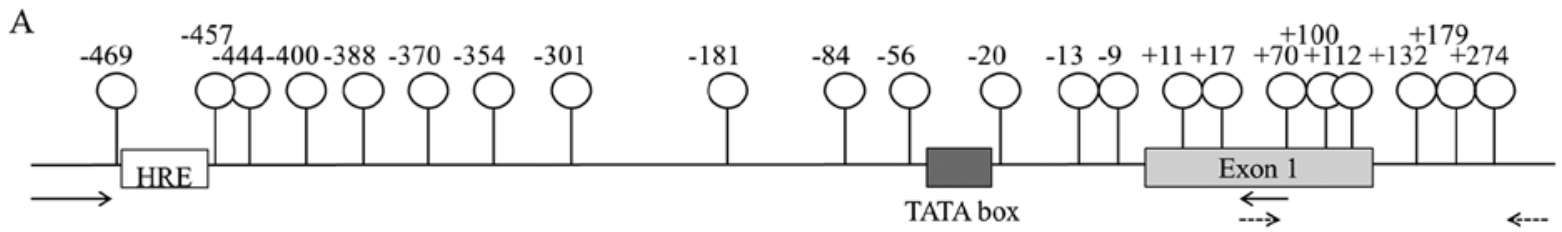

B Cell lines

Low Expression
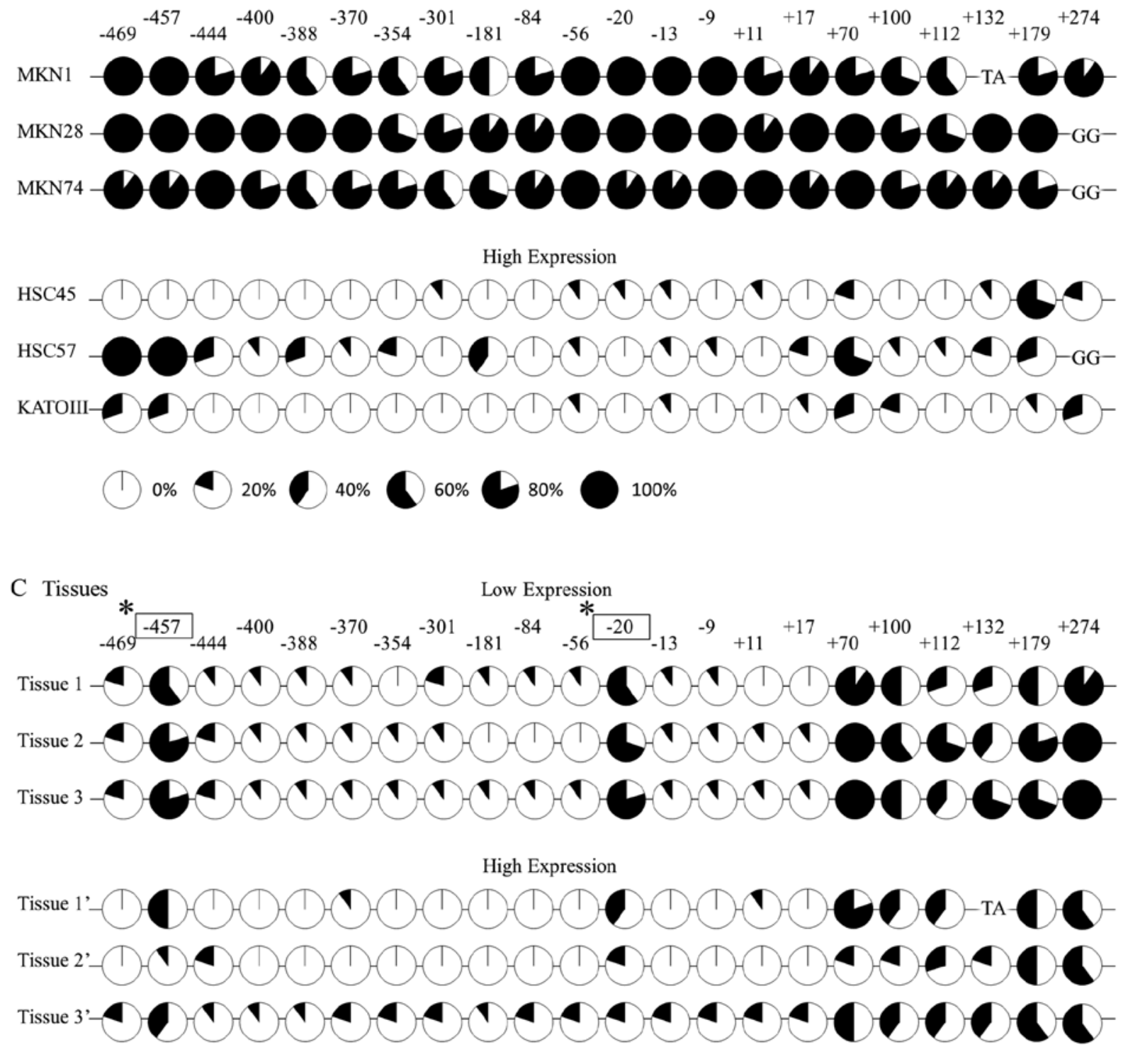

${ }_{0 \%} \bigcirc{ }_{20 \%} \bigcirc{ }_{40 \%} \bigcirc{ }_{100 \%}$

Figure 3. (A) A diagram of the promoter region of the TFF1 gene. Twenty-two of CpG dinucleotides located between 274 bp and 469 bp are shown by 0 . HRE: a consensus sequence of hypoxia response element. Location of bisulfite PCR primers was shown by arrows and dashed arrows. DNA methylation status at $22 \mathrm{CpG}$ sites in the promoter region of TFF1 gene in (B) six gastric cancer cell lines and (C) six resected tissue samples. Circle graphs indicate the methylation rate of each $\mathrm{CpG}$ site. An unmethylated $\mathrm{CpG}$ is shown in white and a methylated GpG is shown in black. The CpG site at +274 bp HSC57, MKN28 and MKN74 cells mutated to 'GG' and the CpG site at +132 bp in MKN1 mutated to 'TA'. The CpG site at +132 bp in Tissue 1 mutated to 'TA'. ${ }^{*}$ The difference in methylation rate is statistically significant $(\mathrm{p}<0.05)$.

a significant correlation between the depth of cancer invasion and the immunohistochemical expression of TFF1 in resected tissue samples from patients with gastric cancer $(\mathrm{p}=0.037$, Table I). Furthermore, in an in vitro analysis using TFF1 knockdown cells established from HSC45 and HSC57, the invasive activity of gastric cancer cells increased signifi- 

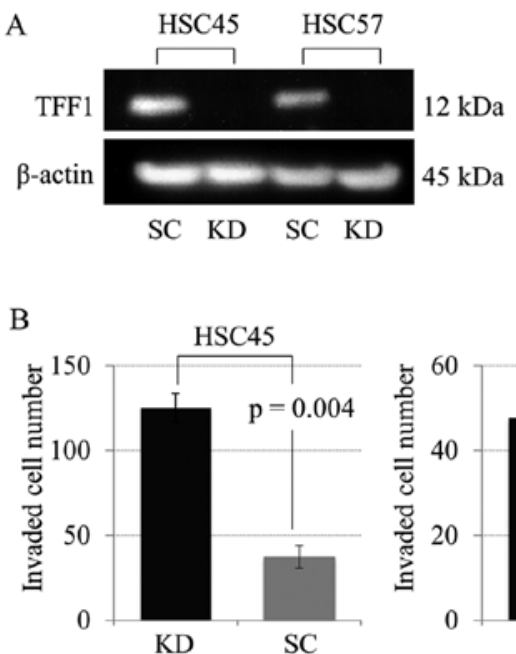

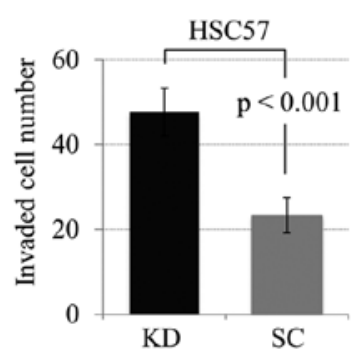

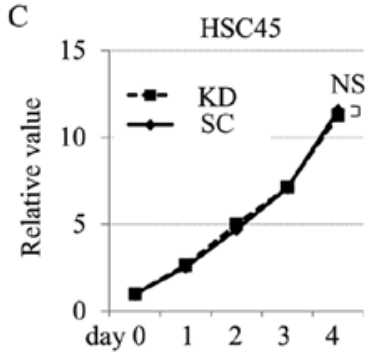

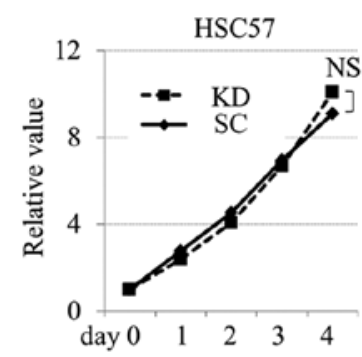

Figure 4. Characterization of HIF-1 $\alpha$ knockdown cells. (A) Western blot analysis of TFF1. The TFF1 expression was detected in the control cells (HSC45-SC and HSC57-SC), but not in TFF1 knockdown cells (HSC45-KD and HSC57-KD). (B) Invasion capacity of the four cell lines was analyzed by an in vitro invasion assay. In both of HSC45 and HSC57 cells, TFF1 knockdown cells exhibited a significantly higher capacity for invasion compared with the control cells (HSC45-KD vs. HSC45-SC $=125.3 \pm 8.5$ vs. $37.3 \pm 6.7$; $\mathrm{p}<0.001$, HSC57-KD vs. HSC57-SC $=47.7 \pm 5.7$ vs. 23.3 $\pm 4.2 ; \mathrm{p}=0.004$ ). (C) MTT proliferation assay was carried out and the viability of the cells was assessed in triplicate experiments on days $0,1,2,3$ and 4 . There was no significant difference in the relative proliferation values on day 4 between the TFF1 knockdown and control cells (HSC45-KD vs. HSC45-SC $=11.29 \pm 0.001$ vs. $11.41 \pm 0.022 ; \mathrm{p}=0.303$, HSC $57-\mathrm{KD}$ vs. HSC57-SC $=9.70 \pm 0.932$ vs. $9.05 \pm 0.235 ; \mathrm{p}=0.378$ ).

cantly in TFF1-deficient cells compared with the control cells (Fig. 4B). These results indicated that TFF1 acts not only as a tumor suppressor gene during gastric carcinogenesis, but also as an inhibitor of tumor invasion during gastric cancer progression. Hence, it is speculated that loss of TFF1 at carcinogenesis might sequentially promote cancer invasion in the stomach.

In a series of survival analysis, low expression of TFF1 tended to be associated with a worse survival $(p=0.078$, Fig. 1C) in all the patients. Furthermore, the expression of TFF1 was an independent predictive factor of patient survival in the surgery group ( $p=0.006$, Fig. 1D). On the contrary, TFF1 expression did not contribute to the survival of patients who received adjuvant therapy $(\mathrm{p}=0.732$, Fig. $1 \mathrm{E})$. One of the reasons for these results might be due to the differences in lymph node metastasis between the two groups. In this series, 67 of 74 patients $(90.5 \%)$ in the adjuvant group had lymph node metastasis, whereas only 50 patients $(46.3 \%)$ in the surgery group had metastatic lymph nodes. TFF1 expression might not influence lymph node metastasis during gastric cancer progression and therefore resulted in no correlation with survival in the adjuvant group, with high level of lymph node metastasis, compared with the surgery group. Furthermore, in vitro analysis revealed no effect of TFF1 expression on the drug sensitivity to 5-FU (data not shown). Taken together, it is suggested that TFF1 expression is a suitable biomarker predicting tumor invasion of gastric cancer.

TFF peptides have been reported to stimulate the migration of epithelial cells to promote the repair of mucosal wounds $(2,7,8)$. Moreover, the oral intake of recombinant human TFF peptides protects the gastric mucosa against ethanol- and indomethacin-induced mucosal injuries in rats (24). Transgenic mice that overexpress human TFF1 in the jejunum demonstrated an increased resistance to indomethacin-induced mucosal damage (25). Clinically, phase II studies have currently demonstrated that oral spray formulation of recombinant human TFF prevented chemotherapy induced oral mucositis in patients with colorectal cancer (26). Based on these studies, oral intake or local administration of TFF1 peptide might suppress tumor invasion of gastric cancer.

The present study further addressed the regulatory mechanism of TFF1 expression. Previous studies have demonstrated the genetic regulation of TFF1, in which the loss of TFF1 expression in gastric cancer was explained by $\mathrm{LOH}$ and mutations (27-31). Yio et al demonstrated that TFF1 mutations enhance the invasion of gastric cancer cells in vitro (31). Park et al also indicated that the allelic loss of TFF1 plays an important role in gastric carcinogenesis and detected somatic missense mutations of TFF1 in 7 of 43 gastric cancers $(27,28)$. In addition to these studies, a low-oxygen environment induced the expression of TFF1 in gastric epithelial cells via the HIF-1 pathway (19). The present study investigated the epigenetic regulation of this gene by means of bisulfate sequencing. In the experiment using six gastric cancer cell lines, the cells with low TFF1 expression showed dense methylation in the promoter region of this gene, while those with high expression levels revealed sparse methylation (Fig. 3B). These results indicate that TFF1 expression in gastric cancer cells is strongly dependent on the DNA methylation of the promoter region. The same experiment approached the methylation status in the resected cancer tissues. Interestingly, cancer tissues with the low expression showed a significantly higher methylation at two specific CpG sites, located at -20 and -457 from the transcript initiation site, compared to the high-expression tissues (Fig. 3C). The result indicated that TFF1 expression is dependent on the site-specific methylation in gastric cancer 
tissues. A TATA box (5'-tataaaa-3') is located upstream of the -20 bp $\mathrm{CpG}$ site and a consensus sequence of hypoxia response element [HRE (5'-gCGtg-3')] is located upstream of the -457 bp $\mathrm{CpG}$ site.

A TATA box is one of the core elements in a promoter region, which is necessary to direct the initiation of transcription, cooperatively acting with other molecules (32). Thus, hypermethylation in the $\mathrm{CpG}$ site at $-20 \mathrm{bp}$ downstream of a TATA box might inhibit the binding of basal transcription complex, resulting in suppression of the basal expression of TFF1. HRE is a binding site of the HIF-1 complex, which induces the expression of hypoxia-target genes under hypoxic conditions (33). In the present study, TFF1 expression was induced by a hypoxic environment in KATO-III and HSC45 cells, whereas little induction was observed in the HSC57 cells (Fig. 2B). The CpG site of -457 bp in the HSC57 cells showed dense methylation in bisulfite sequencing analysis, thus we speculated that epigenetic modifications at this region possibly inhibited the binding of HIF-1 to HRE and thus led to the decreased hypoxic induction in HSC57 cells. Methylation status of these $\mathrm{CpG}$ sites could potentially be a promising biomarker predicting TFF1 expression in gastric cancer tissues.

In conclusion, this is the first report demonstrating that a low expression of TFF1 in gastric cancer is not only associated with the depth of tumor invasion, but also with the poor survival in the patients who undergo curative surgery without adjuvant therapy. Furthermore, epigenetic analysis revealed that TFF1 expression in gastric cancer tissues is significantly dependent on DNA methylation of two specific CpGs at $-20 \mathrm{bp}$ and $-457 \mathrm{bp}$ in the promoter region. The expression or methylation status of TFF1 in gastric cancer tissues might therefore be useful marker predicting patient survival. Moreover, a novel treatment by TFF1 peptide should be selectively performed in gastric cancer patients with low TFF1 expression to improve the survival by inhibiting tumor invasion.

\section{Acknowledgements}

This study was supported by a Grant-in-Aid for Young Scientists (B) (23791548) from the Japan Society for the Promotion of Science. We also thank Mr. Fumihiro Mutoh for his helpful contributions to cutting the embedded frozen specimens for the analyses.

\section{References}

1. Wong WM, Poulsom R and Wright NA: Trefoil peptides. Gut 44: 890-895, 1999

2. Hoffmann W and Jagla W: Cell type specific expression of secretory TFF peptides: colocalization with mucins and synthesis in the brain. Int Rev Cytol 213: 147-181, 2002.

3. Taupin D and Podolsky DK: Trefoil factors: initiators of mucosal healing. Nat Rev Mol Cell Biol 4: 721-732, 2003.

4. Thim L: Trefoil peptides: from structure to function. Cell Mol Life Sci 53: 888-903, 1997.

5. Masiakowski P, Breathnach R, Bloch J, Gannon F, Krust A and Chambon P: Cloning of cDNA sequences of hormone-regulated genes from the MCF-7 human breast cancer cell line. Nucleic Acids Res 10: 7895-7903, 1982.

6. Rio MC, Bellocq JP, Daniel JY, et al: Breast cancer-associated pS2 protein: synthesis and secretion by normal stomach mucosa. Science 241: 705-708, 1988.
7. Tomasetto C, Masson R, Linares JL, Wendling C, Lefebvre O, Chenard MP and Rio MC: pS2/TFF1 interacts directly with the VWFC cysteine-rich domains of mucins. Gastroenterology 118: 70-80, 2000.

8. Hoffmann W, Jagla W and Wiede A: Molecular medicine of TFF-peptides: from gut to brain. Histol Histopathol 16: 319-334, 2001.

9. Lefebvre O, Chenard MP, Masson R, et al: Gastric mucosa abnormalities and tumorigenesis in mice lacking the $\mathrm{pS} 2$ trefoil protein. Science 274: 259-262, 1996.

10. Colombel M, Dante R, Bouvier R, Ribieras S, Pangaud C, Marechal JM and Lasne Y: Differential RNA expression of the pS2 gene in the human benign and malignant prostatic tissue. J Urol 162: 927-930, 1999.

11. Buache E, Etique N, Alpy F, et al: Deficiency in trefoil factor 1 (TFF1) increases tumorigenicity of human breast cancer cells and mammary tumor development in TFF1-knockout mice. Oncogene 30: 3261-3273, 2011.

12. Arumugam T, Brandt W, Ramachandran V, et al: Trefoil factor 1 stimulates both pancreatic cancer and stellate cells and increases metastasis. Pancreas 40: 815-822, 2011.

13. McChesney PA, Aiyar SE, Lee OJ, Zaika A, Moskaluk C, Li R and El-Rifai W: Cofactor of BRCA1: a novel transcription factor regulator in upper gastrointestinal adenocarcinomas. Cancer Res 66: 1346-1353, 2006.

14. Milne AN, Carvalho R, Morsink FM, Musler AR, de Leng WW, Ristimäki A and Offerhaus GJ: Early-onset gastric cancers have a different molecular expression profile than conventional gastric cancers. Mod Pathol 19: 564-572, 2006.

15. Gött P, Beck S, Machado JC, Carneiro F, Schmitt H and Blin N: Human trefoil peptides: genomic structure in 21q22.3 and coordinated expression. Eur J Hum Genet 4: 308-315, 1996.

16. Beck S, Sommer P, Blin N and Gött P: 5'-flanking motifs control cell-specific expression of trefoil factor genes (TFF). Int J Mol Med 2: 353-361, 1998.

17. Al-azzeh ED, Fegert P, Blin N and Gött P: Transcription factor GATA- 6 activates expression of gastroprotective trefoil genes TFF1 and TFF2. Biochim Biophys Acta 1490: 324-332, 2000.

18. Beck S, Sommer P, dos Santos Silva E, Blin N and Gött P: Hepatocyte nuclear factor 3 (winged helix domain) activates trefoil factor gene TFF1 through a binding motif adjacent to the TATAA box. DNA Cell Biol 18: 157-164, 1999.

19. Hernández $\mathrm{C}$, Santamatilde $\mathrm{E}, \mathrm{McCreath} \mathrm{KJ}$, et al: Induction of trefoil factor (TFF) 1, TFF2 and TFF3 by hypoxia is mediated by hypoxia inducible factor-1: implications for gastric mucosal healing. Br J Pharmacol 156: 262-272, 2009.

20. Japanese Gastric Cancer Association: Japanese classification of gastric carcinoma: 3rd English ed. Gastric Cancer 14: 101-112, 2011.

21. Yanagihara K, Ito A, Toge T and Numoto M: Antiproliferative effects of isoflavones on human cancer cell lines established from the gastrointestinal tract. Cancer Res 53: 5815-5821, 1993.

22. Yanagihara K, Tanaka H, Takigahira M, et al: Establishment of two cell lines from human gastric scirrhous carcinoma that possess the potential to metastasize spontaneously in nude mice. Cancer Sci 95: 575-582, 2004.

23. Nakamura J, Kitajima Y, Kai K, et al: Expression of hypoxic marker CA IX is regulated by site-specific DNA methylation and is associated with the histology of gastric cancer. Am J Pathol 178: 515-524, 2011.

24. Babyatsky MW, deBeaumont M, Thim L and Podolsky DK: Oral trefoil peptides protect against ethanol- and indomethacininduced gastric injury in rats. Gastroenterology 110: 489-497, 1996.

25. Playford RJ, Marchbank T, Goodlad RA, Chinery RA, Poulsom R and Hanby AM: Transgenic mice that overexpress the human trefoil peptide $\mathrm{pS} 2$ have an increased resistance to intestinal damage. Proc Natl Acad Sci USA 93: 2137-2142, 1996.

26. Peterson DE, Barker NP, Akhmadullina LI, et al: Phase II, randomized, double-blind, placebo-controlled study of recombinant human intestinal trefoil factor oral spray for prevention of oral mucositis in patients with colorectal cancer who are receiving fluorouracil-based chemotherapy. J Clin Oncol 27: 4333-4338, 2009.

27. Park WS, Oh RR, Park JY, et al: Mapping of a new target region of allelic loss at 21q22 in primary gastric cancers. Cancer Lett 159: 15-21, 2000. 
28. Park WS, Oh RR, Park JY, et al: Somatic mutations of the trefoil factor family 1 gene in gastric cancer. Gastroenterology 119: 691-698, 2000.

29. Carvalho R, Kayademir T, Soares P, et al: Loss of heterozygosity and promoter methylation, but not mutation, may underlie loss of TFF1 in gastric carcinoma. Lab Invest 82: 1319-1326, 2002.

30. Beckler AD, Roche JK, Harper JC, et al: Decreased abundance of trefoil factor 1 transcript in the majority of gastric carcinomas. Cancer 98: 2184-2191, 2003.

31. Yio X, Diamond M, Zhang JY, Weinstein H, Wang LH, Werther L and Itzkowitz S: Trefoil factor family-1 mutations enhance gastric cancer cell invasion through distinct signaling pathways. Gastroenterology 130: 1696-1706, 2006.
32. Lifton RP, Goldberg ML, Karp RW and Hogness DS: The organization of the histone genes in Drosophila melanogaster: functional and evolutionary implications. Cold Spring Harb Symp Quant Biol 42: 1047-1051, 1978.

33. Semenza GL, Jiang BH, Leung SW, Passantino R, Concordet JP, Maire P and Giallongo A: Hypoxia response elements in the aldolase A, enolase 1, and lactate dehydrogenase A gene promoters contain essential binding sites for hypoxia-inducible factor 1. J Biol Chem 271: 32529-32537, 1996. 